Handbuch Frühe Griechische Philosophie 


\section{Handbuch \\ Frühe Griechische Philosophie}

Von Thales bis zu den Sophisten

Herausgegeben von A. A. Long

Aus dem Englischen von Karlheinz Hülser

Verlag J.B. Metzler

Stuttgart $\cdot$ Weimar 
Titel der Originalpublikation: The Cambridge Companion to Early Greek Philosophy, edited by A. A. Long. (C) Cambridge University Press 1999

Abbildung auf dem Umschlag aus: Alain Martin/Oliver Primavesi, L'Empédocle de Strasbourg (P. Strasb. gr. Inv. I665-I666). Introduction, édition et commentaire. Bibliothèque Nationale et Universitaire de Strasbourg und Walter de Gruyter, I999, Planche V

Die Deutsche Bibliothek - CIP-Einheitsaufnahme

\section{Handbuch frühe griechische Philosophie :}

Von Thales bis zu den Sophisten / hrsg. von A. A. Long.

Aus dem Engl. von Karlheinz Hülser.

- Stuttgart; Weimar : Metzler, 200 I.

ISBN 978-3-476-01852-6

ISBN 978-3-476-04370-2 (eBook)

DOI 10.1007/978-3-476-04370-2

Gedruckt auf säure- und chlorfreiem, alterungsbeständigem Papier.

Dieses Werk einschließlich aller seiner Teile ist urheberrechtlich geschützt. Jede Verwertung außerhalb der engen Grenzen der Urheberrechtsgesetzes ist ohne Zustimmung des Verlages unzulässig und strafbar. Das gilt insbesondere für Vervielfältigungen, Übersetzungen,

Mikroverfilmungen und die Einspeicherung und Verarbeitung in elektronischen Systemen.

(C) 2001 Springer-Verlag GmbH Deutschland

Ursprünglich erschienen bei J.B Metzlersehe Verlagsbuchhandlung

und earl Ernst Poeschel Verlag GmbH in Stuttgart 2001

www. metzlerverlag. de

info@metzlerverlag.de

Einbandgestaltung: Willy Löffelhardt

Satz: pagina $\mathrm{GmbH}$, Tübingen

November/200I

Verlag J. B. Metzler Stuttgart · Weimar 


\section{Inhaltsverzeichnis}

Die Autorinnen und Autoren $\ldots \ldots \ldots \ldots \ldots \ldots \ldots \ldots$ VII

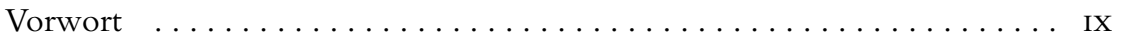

Abkürzungen für Quellen $\ldots \ldots \ldots \ldots \ldots \ldots \ldots \ldots \ldots \ldots \ldots \ldots \ldots$ XIII

Leben und Schriften der frühen griechischen Philosophen $\ldots \ldots \ldots \ldots$ XV

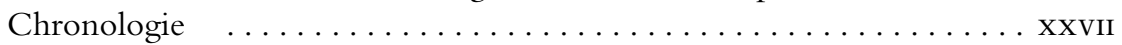

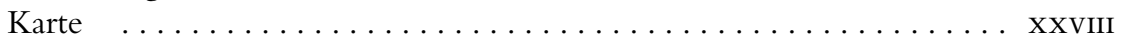

1 Das Anliegen der frühen griechischen Philosophie

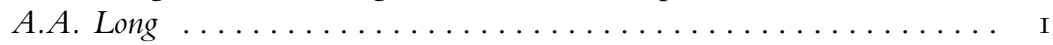

2 Quellen

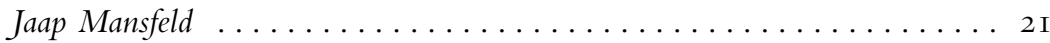

3 Die Anfänge der Kosmologie

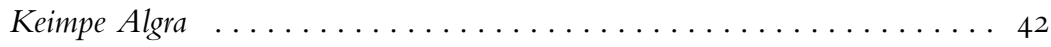

4 Die pythagoreische Tradition

Carl A. Huffman ........................ 6I

5 Heraklit

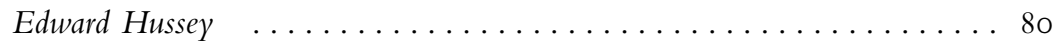

6 Parmenides und Melissos

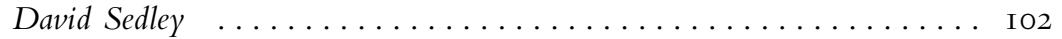

7 Zenon

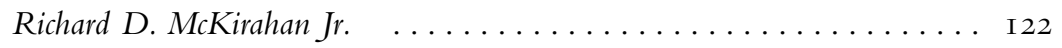

8 Empedokles und Anaxagoras: Antworten auf Parmenides

Daniel W. Graham ..................... I 45

9 Die Atomisten $^{\mathrm{I}}$

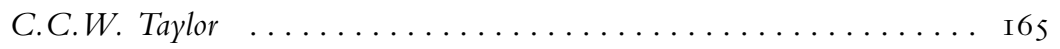

10 Rationale Theologie

Sarah Broadie $\ldots \ldots \ldots \ldots \ldots \ldots \ldots \ldots \ldots \ldots \ldots \ldots \ldots \ldots \ldots \ldots \ldots \ldots$

11 Das frühe Interesse am Wissen

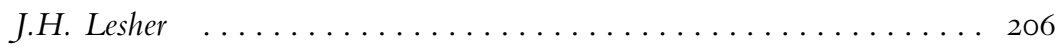

12 Seele, Sinneswahrnehmung und Denken

André Laks . . . . . . . . . . . . . . . . . . . . 228 
13 Schuld, Verantwortung, Ursache: Philosophie, Geschichtsschreibung und Medizin im fünften Jahrhundert

Mario Vegetti .............................. 247

14 Rhetorik und Relativismus: Protagoras und Gorgias

Paul Woodruff .......................... 264

15 Protagoras und Antiphon: Sophistische Erörterungen über Gerechtigkeit

Fernanda Decleva Caizzi ..................... 285

16 Die Poetik der frühen griechischen Philosophie

Glenn W. Most ............................ 304

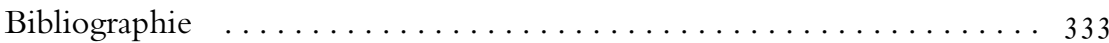

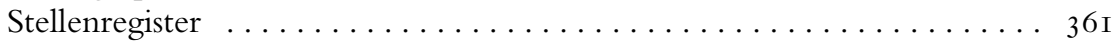

Register der Personen und Sachen $\ldots \ldots \ldots \ldots \ldots \ldots \ldots \ldots \ldots$ 


\section{Die Autorinnen und Autoren}

Keimpe Algra ist Associate Professor für Antike Philosophie an der Universität von Utrecht und Leitender Herausgeber der Zeitschrift Phronesis. Er ist Autor von Concepts of Space in Greek Thought (1995) und Mitherausgeber der Cambridge History of Hellenistic Philosophy (1999).

Sarah Broadie ist Professorin für Philosophie an der Princeton University. Zu ihren Hauptveröffentlichungen gehören Ethics with Aristotle (I99I) und (als Sarah Waterlow) Nature, Change and Agency in Aristotle's Physics (1982).

Fernanda Decleva Caizzi ist Professorin für Antike Philosophie an der Università degli Studi in Mailand und eine der Herausgeber/innen des Corpus dei Papiri Filosofici. Sie ist die Verfasserin von Antisthenis Fragmenta (1965), Antiphontis Tetralogiae (1970), Pirrone testimonianze (I98I), Plato Euthydemus (I996) sowie von Artikeln über philosophische Papyri und über die Traditionen der Sophistik und der Skepsis.

Daniel W. Graham ist Professor für Philosophie an der Brigham Young University in Utah. Geschrieben hat er Aristotle's Two Systems (1987), Aristotle's Physics Book VIII (I995) und zahllose Artikel zur antiken Philosophie.

Carl A. Huffman ist Professor für Classics an der DePauw University in Indiana und Autor von Philolaus of Croton: Pythagorean and Presocratic (1993). Zur Zeit arbeitet er an einer Edition der Fragmente des Archytas von Tarent.

Edward Hussey ist Fellow des All Souls College in Oxford. Er ist Autor von The Presocratics (1972), Aristotle: Physics III-IV (1983) und anderer Veröffentlichungen zur frühen griechischen Philosophie und zu Aristoteles.

André Laks ist Professor für Antike Philosophie an der Universität Charles de Gaulle-Lille 3 in Frankreich. Geschrieben hat er Diogène d'Apollonie (I983) und Artikel zur griechischen Philosophie. Zusammen mit Glenn W. Most hat er Theophrastus Metaphysics (1993) und Studies on the Derveni Papyrus (1997) herausgegeben.

J.H. Lesher ist Professor für Philosophie und Classics an der University of Maryland. Er ist Autor von Xenophanes of Colophon (I992), von The Greek Philosophers (I998) und von zahllosen Studien zu alten griechischen Theorien der Erkenntnis.

A.A. Long ist Professor für Classics und Irving Stone Professor für Literatur an der University of California, Berkeley. Er ist Autor von Language and Thought in 
Sophocles (1968), Hellenistic Philosophy (1974, I986), The Hellenistic Philosophers (zusammen mit David Sedley, I987; dt. Die hellenistischen Philosophen, 2000), Stoic Studies (1996) und verfaßte Artikel zur frühen und späteren griechischen Philosophie.

Richard D. McKirahan Jr. ist E.C. Norton Professor für Classics und Philosophie am Pomona College in Kalifornien. Er ist Autor von Philosophy before Socrates (1994) und Principles and Proofs: Aristotle's Theory ob Demonstrative Science (I992).

Jaap Mansfeld ist Professor für alte und mittelalterliche Philosophie an der Universität Utrecht und hat zur antiken Philosophie zahlreiche Bücher und Beiträge publiziert.

Glenn W. Most ist Professor für Altgriechisch an der Universität Heidelberg und Professor für Social Thought an der University of Chicago. Er ist Autor von The Measures of Praise: Structure and Function in Pindar's Second Pythian and Seventh Nemean Odes (1985), Collecting Fragments - Fragmente sammeln (I997) und von zahlreichen Studien zur antiken und modernen Philosophie und Dichtung. Zusammen mit A. Laks hat er Theophrastus Metaphysics (I993) und Studies on the Derveni Papyrus (1997) herausgegeben.

David Sedley ist Professor für Antike Philosophie an der University of Cambridge und Fellow von Christ's College. Er ist Co-Autor von The Hellenistic Philosophers (zusammen mit A.A. Long, 1987) und Autor von Lucretius and the Transformation of Greek Wisdom (1998).

C.C.W. Taylor ist Reader in Philosophy an der Oxford University und Fellow des Corpus Christi College. Er hat Bücher über Plato und Protagoras geschrieben (I976, I991), über The Greeks on Pleasure (zusammen mit J.C.B. Gosling, I982) und über Socrates (Oxford I998), und zahlreiche Artikeln zur Philosophiegeschichte, zur Ethik und zur Philosophie des Geistes verfaßt.

Mario Vegetti ist Professor für Antike Philosophie an der Universität von Pavia. Er ist Autor von Il coltello e lo stilo (1996), Tra Edipo e Euclide (1983), L'etica degli antichi (I989) und von zahlreichen Werken zur Geschichte der antiken Medizin, Wissenschaft und Philosophie.

Paul Woodruff ist Professor für Philosophie und Thompson Professor of $\mathrm{Hu}-$ manities an der University of Texas in Austin. Er ist Autor von Thucydides on Justice, Power und Human Nature (1993), zusammen mit M. Gagarin Herausgeber von Early Greek thought from Homer to the Sophists (I995) und Übersetzer vieler Platonischer Dialoge sowie der Bakchen des Euripides. 


\section{Vorwort}

Dieses Buch möchte einen neuen und breit angelegten Überblick über die frühe griechische Philosophie bieten und behandelt die Denker, die häufig die Vorsokratiker genannt werden. Die Kapitel sind aufgeteilt in Studien zu einzelnen Denkern oder Bewegungen, einschließlich der Sophisten, und Studien $\mathrm{zu}$ bestimmten Themen, zu denen sie gemeinsam beigetragen haben. Griechisch-Kenntnisse werden nicht vorausgesetzt. Vielmehr enthält das Buch umfangreiche Übersetzungen von Primärtexten, wobei diese Übersetzungen, wenn nichts anderes vermerkt ist, von den Autoren der Beiträge selbst stammen. Am Ende des Bandes findet sich eine detaillierte Bibliographie, die in Übereinstimmung mit den einzelnen Hauptkapiteln angelegt ist. Die Verweise in den Fußnoten auf die Fachliteratur und auf andere Einzelheiten sind in erster Linie mehr dazu gedacht, den gewöhnlichen Leser zu unterstützen, als dazu, Feinabstimmungen vorzunehmen. Abkürzungen von Verweisen auf antike Autoren und ihre Werke werden zu Beginn des Bandes erklärt. Ebenfalls am Anfang findet man eine Karte, die die Geburtsstädte der Philosophen und die Städte zeigt, in denen sie sich niedergelassen haben, ferner eine Liste, die mit Zeitlinien (in der Regel) annäherungsweise die Lebensdaten der Philosophen darstellt, und eine alphabetische Übersicht über deren Biographien und Schriften.

Für diejenigen, die sich der frühen griechischen Philosophie erstmals nähern, werden ein paar beratende Worte zum Gebrauch dieses Buches hilfreich sein. In Kapitel I biete ich einen Überblick über das Feld an, das die Kapitel 3-I6 im einzelnen erkunden. Weil die Belegtexte so fragmentarisch und häufig durch Zusammenfassungen aus zweiter oder dritter Hand überliefert sind, anstatt die eigenen Worte der Denker wiederzugeben, ist es unerläßlich, zumindest in allgemeiner Weise mit den späteren griechischen (gelegentlich auch römischen) Autoren vertraut zu sein, die unsere unmittelbaren Quellen sind. Denjenigen, die sich dem Gegenstand neu zuwenden, sei deshalb dringend geraten, Kapitel 2 zu lesen, die Studie von Jaap Mansfeld über die Quellen, bevor sie mit einem der anschließenden Beiträge fortfahren; dieses Kapitel ist außerdem die passende Stelle, um sich Hilfe zu alten Verweisen im Haupttext und in den Fußnoten zu holen. Das übrige Buch ist so gestaltet, daß man es der Reihe nach lesen kann. Die Kapitel sind aber alle in sich geschlossen, und es werden keine Voraussetzungen hinsichtlich der Reihenfolge gemacht, in der man sie liest. Diejenigen, deren erstes Interesse den Sophisten gilt, könnten sich sofort den Kapiteln I4 und 15 zuwenden. An die thematischen Kapitel kann man sich gleichfalls in jeder beliebigen Reihenfolge machen. Freilich werden Leser, die mit den in 
den Kapiteln 3-9 erörterten Philosophen nicht vertraut sind, es vorziehen, diese chronologisch angeordneten Untersuchungen $\mathrm{zu}$ Einzelpersönlichkeiten und Bewegungen zu lesen, bevor sie sich an die Mehrzahl der thematischen Kapitel heranmachen. Abgesehen davon ist das letzte Kapitel, Glenn Mosts Studie zur "Poetik", zwar thematisch ausgerichtet; es befaßt sich aber mit einem Gebiet, das für das Buch als ganzes hoch relevant ist; es läßt sich ebenso als Abschluß wie als eine komplementäre Ergänzung zu meinem Einleitungskapitel lesen.

Alle, die zu diesem Buch beigetragen haben, hatten innerhalb der Umfangsgrenzen vollkommen freie Hand, ihren Gegenstand so zu präsentieren, wie sie es als passend ansahen. Sie wurden weder darum gebeten, orthodox zu sein oder in konventioneller Weise zu verfahren (als ob Orthodoxie und Konventionalität in dieser oder irgendeiner anderen Geschichte Geltung beanspruchen könnten), noch darum, um Originalität zu ringen, sondern lediglich darum, handbuchtauglich zu sein. Nichts kann natürlich die unmittelbare Begegnung ersetzen, die jeder ernsthafte Student mit den Primärtexten suchen wird. Wie wir hoffen, wird dieses Buch seine Benutzer aber zu Punkten von zentralem Interesse führen, ohne allzu grobe Vereinfachungen vorzunehmen oder Barrieren gelehrten Wirrwarrs zu errichten. Wir würden uns freuen, wenn unsere Leser viele der hier vorgetragenen Ideen schwierig finden würden: Die frühe griechische Philosophie würde nicht so intensiv studiert, wenn sie leicht wäre; und je mehr man sie studiert, um so schwieriger wird sie. Weniger erfreut wären wir, wenn unsere Darstellungen für schwierig gehalten werden sollten und wenn der Anreiz in bezug auf das Material nicht im Verhältnis zu den Schwierigkeiten wächs, die man beim Nachdenken darüber erfährt. Wenn einer sich selbst findet, wie er mit Heraklit, Parmenides oder Zenon debattiert oder mit dem auseinandersetzt, was unsere Autoren über diese und andere Dinge sagen, dann ist es so, wie es sein sollte. Es wird nie eine abschließende oder gar eine ganz erschöpfende Interpretation der frühen griechischen Philosophen geben, und in diesem Buch lassen sich durchaus (worauf ich gelegentlich hingedeutet habe) bei vielen Hauptpunkten unterschiedliche Einschätzungen finden. Sich dem Material aus einer neuen Perspektive zu nähern ist immer möglich; und von Zeit zu Zeit wird das, wovon wir dachten, wir wüßten es, durch bemerkenswerte Entdekkungen erschüttert, so durch den Derveni-Papyrus und ganz vor kurzem durch einen Papyrus, der neue Zeilen von Empedokles enthält. (Für den DerveniPapyrus siehe Most, in diesem Band S. 3 I2, und Laks \& Most [537]. Das neue Empedokles-Material wurde I998 durch Martin \& Primavesi [380] veröffentlicht.)

Es erscheinen ständig Ergebnisse neuer Forschung zur frühen griechischen Philosophie. (Einen hilfreichen Überblick über neuere Trends in der Forschung gibt Mourelatos [155] XXI-XXVII.) Die Bibliographie dieses Buchs ist zwar lang, mußte aber trotzdem ganz selektiv vorgehen; und sie enthält Titel, die zu neu waren, um durchgehend berücksichtigt und gewürdigt zu werden. Dazu gehört Peter Kingsleys herausforderndes Werk über Empedokles und die pythagoreische Tradition [105], das ganz neue Ideen entwickelt, eine Verbindung der frühen griechischen Philosophie zur Magie herstellt und deren Überliefe- 
rung nach Ägypten und zum Islam sowie zur mittelalterlichen Mystik und zur Alchemie hin nachzeichnet. Als dieses Handbuch in der letzten Phase seiner Entstehung war, erschien das wichtige Buch von Patricia Curd, The Legacy of Parmenides [290], und noch ein weiteres Buch von Kingsley, In the Dark Places of Wisdom (Inverness, California, I999), welches im Licht von Inschriften, die man in Velia in Süditalien entdeckt hat, Parmenides neu interpretiert. (Für Einzelheiten dieser Inschriften siehe Coxon [270] 39-40.) Untersuchungen wie diese bestärken uns in der Erwartung, daß die frühe griechische Philosophie im einundzwanzigsten Jahrhundert das Denken und die Neuinterpretation ebenso stimulieren wird wie in den letzten hundert Jahren.

Das Buch zustandezubringen hat länger gedauert, als ich es vorausgesehen habe, als ich die Einladung von Terry Moore, dem Herausgeber der Companion-Reihe bei Cambridge University Press, annahm, der Herausgeber dieses Handbuchs zur frühen griechischen Philosophie zu sein. Ihm und allen meinen Mitautoren möchte ich für ihre Geduld und für ihre großartige Kooperation danken. Besonders dankbar bin ich Keimpe Algra, dem Autor von Kapitel 3, der diese Arbeit kurzfristig übernahm, nachdem ein zunächst vorgesehener Mitautor mit seinem Beitrag nicht weiterkam. Das moderne Studium der frühen griechischen Philosophie ist lange ein attraktives internationales Unternehmen gewesen. Ich bin besonders erfreut, daß die Autoren des Buchs fünf Nationalitäten vertreten und den Universitäten von sechs Ländern affiliiert sind.

Während der Arbeit an der Herausgabe des Bandes stand mir James Ker zur Seite, graduierter Classics-Student in Berkeley. Er war mir eine unschätzbare Hilfe bei der Zusammenstellung der Bibliographie und von anderem Ergänzungsmaterial, beim Formatieren der Kapitel und beim Aufspüren von Verweisen. Von all dem abgesehen kamen mir seine Begeisterung zugute, seine fruchtbaren Anregungen und seine Bereitschaft, sich selbst in die Lage von jemand zu versetzen, der das Buch benutzt.

Mein eigenes Studium der frühen griechischen Philosophie begann am University College in London unter der großartigen Anleitung David Furleys. Vierzig Jahre später auf jene Zeit zurückblickend, sehe ich, daß Heraklit, Parmenides und die anderen frühen griechischen Philosophen der Hauptgrund waren, weswegen ich mich in die antike Philosophie verliebt und die Philosophie überhaupt schätzen gelernt habe. Dieses Buch wird seinen Zweck dann erfüllen, wenn es anderen dabei hilft, solch eine Anziehung zu erfahren. 


\section{Abkürzungen für Quellen}

Fragmente werden nach der Sammlung von Diels/Kranz [I] zitiert. Die Angabe "DK 28 B6,4-7《 zum Beispiel bezieht sich auf die Zeilen 4-7 von Fragment B6 des Parmenides, dessen Autor-Nummer in DK die Nummer 28 ist. (Zu der A/B-Unterscheidung siehe Mansfeld S. 23f.)

$\mathrm{Zu}$ modernen Werken, die mit einer Nummer in eckigen Klammern zitiert werden (z.B. "Barnes [I4]«), findet man die vollständigen bibliographischen Angaben in der Bibliographie. Eine Liste mit Abkürzungen für Zeitschriften ist auf S. 334 beigefügt.

$\begin{array}{ll}\text { Adv. Col. } & \text { Plutarch, Gegen Kolotes (Adversus Colotem) } \\ \text { Anc. Med. } & \text { [Hippokrates], Über die alte Medizin } \\ \text { APo } & \text { Aristoteles, Analytica posteriora } \\ \text { Apol. } & \text { Platon, Verteidigungsrede des Sokrates (Apologia) } \\ \text { Cat. } & \text { Aristoteles, Kategorien } \\ \text { De an. } & \text { Aristoteles, Über die Seele (De anima) } \\ \text { DK } & \text { Diels/Kranz, Die Fragmente der Vorsokratiker [I] } \\ \text { D.L. } & \text { Diogenes Laertius } \\ \text { FHSG } & \text { Fortenbaugh/Huby/Sharples/Gutas, Theophrastus of Ere- } \\ & \text { sus. Sources for his Life, Writings, Thought and Influence [37] } \\ \text { GA } & \text { Aristoteles, Über die Entstehung von Lebewesen (De gene- } \\ & \text { ratione animalium) } \\ \text { GC } & \text { Aristoteles, Über Werden und Vergehen (De generatione et } \\ & \text { corruptione) } \\ \text { Gorg. } & \text { Platon, Gorgias } \\ \text { Il. } & \text { Homer, Ilias } \\ \text { In phys., In Parm. etc. } & \text { Kommentar zu Aristoteles' Physik, Kommentar zu Platons } \\ & \text { Parmenides etc. } \\ \text { Krat. } & \text { Platon, Kratylos } \\ \text { KRS } & \text { Kirk/Raven/Schofield, The Presocratic Philosophers [4] } \\ & \text { bzw. deutsch: Die vorsokratischen Philosophen } \\ \text { LSJ } & \text { Liddell, H.C., \& Scott, R.A., Greek-English Lexicon, rev. } \\ \text { M. } & \text { H.S. Jones, 9. Aufl. mit Suppl. (Oxford I968) } \\ \text { Metaph. } & \text { Sextus Empiricus, Gegen die Wissenschaftler (Adversus ma- } \\ \text { Meteor. } & \text { thematicos) } \\ \text { Mem. } & \text { Aristoteles, Metaphysik; Theophrast, Metaphysik } \\ & \text { Aristoteles, Meteorologie } \\ & \text { Xenophon, Memorabilien }\end{array}$


MXG

Nat. hom.

$N E$

Od.

Parm.

PH

Phys.

Prot.

Ref.

SE

Sens.

Soph.

Theog.

Tht.

VS
[Aristoteles], Über Melissos, Xenophanes, Gorgias

[Hippokrates], Über die Natur des Menschen (De natura hominis)

Aristoteles, Nikomachische Ethik

Homer, Odyssee

Platon, Parmenides

Sextus Empiricus, Grundriß der pyrrhonischen Skepsis (Pyrrhoneae hypotyposes)

Aristoteles, Physik; Eudemos, Physik

Platon, Protagoras

Hippolytos, Widerlegung aller Häresien (Refutatio omnium haeresium)

Aristoteles, Sophistische Widerlegungen (Sophistici elenchi) Theophrast, Über die Sinne (De sensibus)

Platon, Sophistes

Hesiod, Theogonie

Platon, Theaitet

Philostratos, Leben der Sophisten (Vitae sophistarum) 


\section{Leben und Schriften der frühen griechischen Philosophen}

\section{Anaxagoras}

Geboren ca. 500 v.Chr. in Klazomenai an der jonischen Küste; Autor eine Kosmologie, die alle letzten Elemente irgendwelcher Art verwirft und die den Nous (Geist) als ihr tätiges Prinzip hat. Anaxagoras war der erste Philosoph, der sich in Athen niedergelassen hat, wo er etwa 20 Jahre verbrachte (unter der Schirmherrschaft des Perikles), bis er wegen Gottlosigkeit angeklagt oder verfolgt wurde. Dann verließ er Athen, wahrscheinlich in Richtung Lampsakos, und starb ca. 428 v.Chr. Für eine neuere Rekonstruktion seines Lebenswegs siehe Mansfeld [395].

\section{Quellen}

D.L. II,6-I 5; Suda; Platon, Apol. 26d; Phaedros 270a; Plutarch, Perikles 6, I6, 32; weitere Quellen bei DK 59 A.

\section{Werke}

"Eine einzige Schrift« (D.L. I,I6), die später unter dem Titel Über die Natur bekannt war und sich über zwei Bücher erstreckte. Sechzehn Passagen ihres "ersten Buchs" (darunter die Anfangsworte "Zusammen waren alle Dinge«) werden von Simplikios zitiert, und bis auf eine finden wir sie alle in dessen Kommentar zur Physik des Aristoteles; andere Autoren haben uns einige wenige zusätzliche Zeilen überliefert. Andere Bücher, die ihm zugeschrieben wurden (über die Quadratur des Kreises, über Bühnenmalerei und Perspektive sowie über Probleme (DK 59 A38-40), waren so gut wie sicher unecht.

\section{Anaximander}

Geboren ca. 6ro v.Chr. in Milet; der früheste Denker, von dem eine detaillierte Kosmologie bezeugt ist. Anaximander soll den Gnomon erfunden und dann in Sparta die erste griechische Sonnenuhr aufgestellt haben; weiter soll er als erster eine Karte der bekannten Welt gezeichnet und ein astronomisches Modell des Himmels gebaut haben. Er starb ca. 546 v.Chr. 
Quellen

D.L. II, I-2; Suda; weiter Quellen bei DK I2 A.

Werke

Anaximander war einer der ersten Griechen, die ein Buch in Prosa verfaßten. Außer daß er die Kosmogonie und Kosmologie erörterte, machte er sich darin auch Gedanken über die Ursprünge des menschlichen Lebens. Die Suda verzeichnet als seine Werke: „Über die Natur, Der Umfang der Erde, Über die Fixsterne, Himmelsglobus und einige andere«. Diese Spezifikationen passen zwar zu den von ihm bekannten Studien, sind aber wohl anachronistische Beschreibungen eines ursprünglich unbetitelten Werks. Für den einzigen vollständigen Satz, der davon erhalten ist, siehe Algra, in diesem Band S. 52.

\section{Anaximenes}

Geboren in Milet; ein jüngerer Zeitgenosse des Anaximander, setzte die milesische Kosmologie fort. Seine Blütezeit war ungefähr 546-526 v.Chr.

\section{Quellen}

D.L. II,3; Suda; weitere Quellen bei DK I 3 A.

Werke

Diogenes Laertius vermerkt, daß Anaximenes "einfach und ungekünstelt « schrieb (II,3). Für Beispiele seiner lebendigen Phraseologie siehe Most, in diesem Band S. 32 If.

\section{Antiphon}

Athenischer Sophist des 5. Jahrhunderts v.Chr., der zwischen natürlicher und gesetzlicher bzw. konventioneller Gerechtigkeit unterschied; vielleicht (wie dies in diesem Band vorgeschlagen wird, siehe Caizzi, S. 302 Anm. 9) identisch mit Antiphon von Rhamnus, dem Attischen Redner (ca. 480-4I I v.Chr.), der dabei half, die oligarchische Revolution von 4I I zu planen, und der deswegen hingerichtet wurde.

\section{Quellen}

(I) Für Antiphon in der Identität des "Sophisten«: Xenophon, Mem. I,6, I-5. IO-I 5; sonstige Quellen bei DK 87 A. (2) Für Antiphon in der Identität des »Redners«: Thukydides VIII,68; Philostratos, VS I, I5. 
Werke

Für Antiphon (I): Über die Wahrheit (teilweise erhalten, siehe Caizzi, in diesem Band Kap. I 5), und die folgenden verlorenen Schriften: Über die Eintracht, Politikos, Über die Traumdeutung. Außerdem gibt es Belege für sein Interesse an Mathematik und Astronomie; siehe DK 87 BI3. Von Antiphon (2) sind verschiedene Reden erhalten, darunter eine Reihe von Tetralogien; dabei handelt es sich um rhetorische Übungen für die Anklage und die Verteidigung eines $\mathrm{Mu}-$ sterfalls (siehe Vegetti, in diesem Band S. 25of.).

\section{Demokrit}

Geboren um 460 v.Chr. in Abdera in Thrakien; Anhänger des Leukipp und Hauptautor des Atomismus. Demokrit war mit Sicherheit mit der eleatischen Philosophie vertraut, und möglicherweise war ihm Anaxagoras bekannt. Er machte ausgedehnte Reisen, wahrscheinlich nach Ägypten und vielleicht sogar nach Indien. In der römischen Welt war er als »der lachende Philosoph« bekannt. Sein Todesdatum kennen wir nicht.

\section{Quellen}

D.L. IX,34-49 (darin enthalten ein Schriftenkatalog); die Suda; weitere Quellen bei DK 68 A.

\section{Werke}

Bei D.L. IX,46-48 sind über 60 Titel bezeugt, die zum größten Teil unter den folgenden Themen eingeordnet sind (die Klassifikation schreibt man Thrasyllos zu, der in Alexandria im frühen I. Jahrhundert n.Chr. Bibliothekar war): Schriften zur Ethik, zur Physik, zur Mathematik, zur Musik (einschließlich der Dichtung) und zu den Künsten. Eine repräsentative Auswahl von Titeln ist: Von der Seelenheiterkeit, Über die Planeten, Von den Farben, Ursachen in betreff des Schalls, Von den irrationalen Linien und Körpern, Von der Poesie und Von der Malerei. Keine der Schriften ist erhalten. Die meisten der bezeugten Fragmente sind ethische Maximen und in der Anthologie des Stobaeus überliefert, der ungefähr I30 Sprüche unter dem Namen Demokrits mitgeteilt hat. Weitere 86 kurze Aphorismen sind in zwei Stobaeus-Handschriften als Die goldenen Sprüche des Philosophen Demokrates aufgelistet. Die Überlieferung davon ist unabhängig von Stobaeus selbst (siehe DK Bd. 2, I 54), und es wurde weithin angenommen, daß Demokrates in Wirklichkeit Demokrit ist. Platon erwähnt ihn niemals namentlich. Unsere beste Quelle für seinen Atomismus ist Aristoteles. 


\section{Diogenes}

Geboren ungefähr 460 v.Chr. in der Milesischen Kolonie Apollonia am Schwarzen Meer, verbrachte Diogenes einige Zeit in Athen. Dort wurde er von Aristophanes in der Komödie Die Wolken verspottet, weil er die göttliche Luft zum ersten und einzigen Prinzip der Welt machte. Diogenes ist sowohl wegen seiner Rückkehr zu einem einzigen Prinzip wichtig als auch deshalb, weil er dieses Prinzip als vernünftig und zielbewußt betrachtete, dies vermutlich unter dem Einfluß von Anaxagoras' Nous. Seine Forschungen galten auch der menschlichen Physiologie und Erkenntnis. Sein Todesdatum ist nicht bekannt.

Quellen

D.L. IX.57; Theophrast, Sens. 39-45; außerdem andere Quellen, die bei DK 64 A zusammengestellt sind.

Werke

Eine Abhandlung Über die Natur, von der ungefähr zehn Fragmente erhalten sind. Die meisten davon hat uns Simplikios aufbewahrt, in seinem Kommentar zur Physik des Aristoteles.

\section{Empedokles}

Geboren etwa 492 v.Chr. in Akragas, Sizilien, in einer angesehenen Familie; Pionier der unermeßlich einflußreichen Theorie von den vier Grundelementen (Erde, Luft, Feuer, Wasser); stand wahrscheinlich in Verbindung zu lokalen Pythagoreern, deren religiöse und ethische Lehren zusammen mit den Argumenten des Parmenides ihn entscheidend beeinflußten. In Akragas unterstützte Empedokles den Übergang von der Tyrannis zur Demokratie. Er wurde bald eine legendäre Gestalt; man schrieb ihm Wundertätigkeit zu, und daß er sein Leben beendet habe, indem er sich in den Krater des Ätna gestürzt habe. Die anekdotenhafte Überlieferung muß sich zum Teil auf die ausgeprägt bizarren Aussagen gründen, die er in seiner Dichtung über sich selbst macht (siehe Most, in diesem Band S. 325f.). Er war aber offenbar eine charismatische Figur, und die Überlieferung, daß er ein Arzt und ein ausgezeichneter Redner gewesen sei, könnte echt sein. Die Verse des Empedokles wurden ins Lateinische übersetzt und dienten als Modell für das große Lehrgedicht des Lukrez, De rerum natura. Empedokles starb ca. 432 v.Chr.

\section{Quellen}

DK 3 г Bir2-I i4; D.L. VIII,5 I-77; die Suda; Aristoteles, Metaph. I,3, 984a I ; weitere Quellen bei DK 3 I A. 
Werke

Empedokles verfaßte Lehrdichtung in Hexametern; sie soll an die 5000 Verse umfaßt haben (D.L. VIII,77) und in ein Werk Über die Natur und ein anderes mit dem Titel Reinigungen (Katharmoi) geteilt gewesen sein. Von den erhaltenen Versen (etwa Iooo Zeilen) wird das meiste im allgemeinen dem ersten dieser beiden Werke zugerechnet; einige Forscher (siehe Osborne [364] und Inwood [357]) denken aber, daß Empedokles nur ein einziges Gedicht geschrieben hat, welches unter beiden Titeln bekannt ist. Dieser Punkt könnte durch den kürzlichen Fund eines Papyrus klarer werden, der bis dahin unbekannte Zeilen enthält (siehe Martin \& Primavesi [380]). Wie es heißt, hat Empedokles außerdem ein kurzes Gedicht zur Medizin, einen Übergang des Xerxes [über den Hellespont], Epigramme und Tragödien geschrieben.

\section{Gorgias}

Geboren ca. 480 v.Chr. in Leontinoi in Sizilien und weithin dafür bekannt, hundert Jahre alt geworden zu sein; ein gefeierter Sophist, besonders geschätzt als Lehrer der Rhetorik. Gorgias besuchte Athen im Jahr 427 als Botschafter. Sein literarischer Stil bevorzugt symmetrisch ausbalancierte, häufig reimende Ausdrucksweisen und war außerordentlich innovativ und einflußreich.

\section{Quellen}

Gorgias ist der namentlich genannte Gegenstand eines der größeren Dialoge Platons. Andere Quellen sind: Philostratos, VS I, I, I,9, I-6; die Suda; Diodorus Siculus XII,53,I-5; weitere Quellen bei DK 82 A.

\section{Werke}

Erhalten sind zwei Reden ganz - das Enkomion der Helena und die Verteidigung des Palamedes - sowie ein Fragment seiner Begräbnisrede. Zusammenfassungen seiner philosophischen Abhandlung Über das Nichtseiende sind erhalten in der pseudo-aristotelischen Schrift Über Melissos, Xenophanes und Gorgias sowie bei Sextus Empiricus, M. 7,65ff. (= $\mathrm{B}_{3}$ ).

\section{Heraklit}

Seine Geburt in Ephesos setzt man allgemein um etwa 540 v.Chr. an und macht ihn damit eine Generation älter als Parmenides. Auch wenn das wahrscheinlich richtig ist, so ist es doch keineswegs sicher. Durchaus mehr als nur ein Gelehrter (siehe Hölscher [I53] I6I) hat die beiden zu Zeitgenossen gemacht und sieht Heraklit eher auf Parmenides antworten als andersherum, wie das im allgemeinen angenommen wird. Heraklits notorisch dunkle Philosophie wurde in populärer Weise durch die Formulierung zusammengefaßt: "Alles ist im Fluß.» 
Von der biographischen Information über seinen misanthropischen Charakter und seine Arroganz ist das meiste von Heraklits eigenen Aussagen abgeleitet. Glaubhaft ist allerdings die Tradition, nach der er sein Recht, die Königswürde zu erben, an seinen Bruder abgetreten habe (D.L. IX,6). Gestorben ist Heraklit wahrscheinlich zwischen 480 und 470 v.Chr.

\section{Quellen}

D.L. IX,I-I7; die Suda; Strabon XIV 632-33, 642; weitere Quellen bei DK $22 \mathrm{~A}$.

\section{Werke}

Es werden mehr als Ioo kurze Aussprüche zitiert, insbesondere von Schriftstellern der christlichen Zeitrechnung. Einige der Aussprüche sind unecht, und der exakte heraklitische Inhalt anderer ist oft schwer $\mathrm{zu}$ bestimmen. Unter der Autorität der Stoiker, deren Philosophie er stark beeinflußte, erwarb Heraklit in der späteren Antike den Status eines Weisen, und zu seinen kryptischen Aussagen wurden zahlreiche Nachahmungen verfaßt (siehe Mondolfo \& Tarán [235]). Seit Aristoteles (Rhetorik III,5, I4O7b 13) bezieht man sich auf "Schriften« Heraklits oder auf ein "Buch" von ihm, das er im Tempel der Artemis in Ephesos niedergelegt haben soll (D.L. IX,5). Es gibt keinen Grund anzunehmen (wie das von Kirk [233] vorgeschlagen wurde), daß Heraklit ein ausschließlich mündlicher Autor gewesen sei. Die Form seiner schriftlichen Äußerungen war aber offenbar mit Absicht epigrammatisch und kryptisch und verzichtete auf die Bindewörter normaler Prosa.

\section{Hippias}

Geboren in der ersten Hälfte des 5. Jahrhunders v.Chr. in Elis auf der Peloponnes, war Hippias der vielseitigste aller Sophisten und berühmt wegen seiner mnemonischen Fähigkeiten. Er war ein "universaler Mann", der in Mathematik, Astronomie, Grammatik, Musik und Geschichte selbständige Forschungen anstellte und der verschiedene poetische Gedichte verfaßte. Außerdem war er der erste, der die Meinungen früherer Schriftsteller sammelte und klassifizierte und der deshalb zum Pionier der doxographischen Tradition wurde (siehe Mansfeld, in diesem Band S. 25). Gestorben ist er wahrscheinlich in den ersten Jahren des 4. Jahrhunderts v.Chr.

\section{Quellen}

Hippias ist der namentlich genannte Gegenstand zweier platonischer Dialoge, von denen aber keiner eine angemessene Vorstellung von seiner Bedeutung vermittelt. Er tritt auch in Platons Protagoras auf. Siehe ferner Philostratos, VS I,I I,I-8; Xenophon, Mem. IV,4; außerdem weitere Quellen bei DK 86 A. 
Werke

Von Hippias' Schriften ist praktisch nichts erhalten; und selbst die wenigen erhaltenen Titel vermitteln kaum ein gerechtes Bild seiner vielseitigen gelehrten Forschungen.

\section{Leukipp}

Geboren in der ersten Hälfte des 5. Jahrhunderts v.Chr. in Milet oder Abdera, war Leukipp "der erste, der Atome als Prinzipien hingestellt hat" (D.L. IX,30). Einzelheiten seines Lebens sind nicht mit Sicherheit bekannt. Man nimmt aber an, daß er später als Parmenides und wohl auch später als Zenon schrieb, dessen Schüler er gewesen sein soll. Sein Todesdatum ist nicht bekannt.

\section{Quellen}

D.L. IX,30-33 und weitere bei DK 67 A.

\section{Werke}

Die Große Weltordnung (eine Schrift, die im Werkverzeichnis Demokrits angeführt wird) wurde von Theophrast Leukipp zugeschrieben (D.L. IX,45). Ein weiteres Werk Über den Geist wird als die Quelle des einen wörtlichen Zitats angegeben, das uns überliefert ist (DK $67 \mathrm{~B} 2)$; siehe dazu Taylor, in diesem Band S. I69.

\section{Melissos}

Geboren im frühen 5. Jahrhundert v.Chr. auf Samos, unterstützte das philosophische Gedicht des Parmenides und arbeitete es in Prosa aus. Staatsmann und Admiral von Samos; 44I4O v.Chr. schlug er die Athener in einer Seeschlacht. Wann er starb, ist nicht bekannt.

\section{Quellen}

D.L. IX,24; die Suda; Plutarch, Perikles 26-28, Themistokles 2.

\section{Werke}

Nach Simplikios (DK 30 A4) ein Buch mit dem Titel Über die Natur oder über das Seiende. Von Simplikios werden daraus acht Textstücke zitiert, bis auf eins alle in seinem Kommentar zur Physik des Aristoteles. Weiteres Material zu Melissos bietet die pseudo-aristotelische Abhandlung Über Melissos, Xenophanes, Gorgias (DK 30 A5). 


\section{Parmenides}

Geboren ca. 5I5 v.Chr. in Elea in Süditalien; der Begründer der eleatischen Philosophie, welche die Wahrheit, die sich über die Wirklichkeit herleiten läßt, einschließlich ihrer Einzigkeit, der trügerischen Vielfalt und Veränderlichkeit der Erscheinungen gegenüberstellt. Parmenides war ein reicher Mann von adliger Geburt. In seiner Jugend hatte er möglicherweise etwas Verbindung zu Xenophanes und sicherlich zu Ameinias, einem Pythagoreer, den er ehrte, indem er ihm ein Heroenheiligtum errichtete. Parmenides soll als Gesetzgeber für Elea tätig geworden sein (Speusipp, Frgm. I) und Athen besucht haben, als er ungefähr 65 Jahre alt war (Platon, Parm. I27b); Platons Chronologie ist allerdings suspekt: siehe Mansfeld [32] 64-68). Parmenides starb in dem Zeitraum von etwa $449-440$ v.Chr.

\section{Quellen}

D.L. IX,2I-23; die Suda; Platon, Parm. I27a-c; weitere Quellen bei Coxon [270].

\section{Werke}

Ein Hexameter-Gedicht, wovon I 54 Zeilen erhalten sind, das längste zusammenhängende Stück durch ein einziges Zitat des Simplikios in seinem Kommentar zur Physik des Aristoteles (144,26). Das Werk hatte drei Teile: ein Proömium von 32 Zeilen (bis auf die letzten zwei alle von Sextus Empiricus, $M$. VII, I I Iff., zitiert); der Weg der Wahrheit (72 Zeilen erhalten, vielleicht neun Zehntel des Originals); schließlich der Weg der Meinungen (44 vollständige Zeilen bezeugt, 6 in einer lateinischen Übersetzung von Caelius Aurelianus). $\mathrm{Zu}$ der argumentativen Beziehung, die diese Teile zueinander haben, siehe Sedley, in diesem Band S. I I2f. Das ganze Gedicht trug in der späteren Antike den Titel Über die Natur.

\section{Philolaos}

Geboren ca. 470 v.Chr. in Kroton (oder Tarent) in Süditalien; der früheste pythagoreische Philosoph, von dem Schriften erhalten sind. In Platons Phaidon (63c) behaupten die Thebanischen Gesprächspartner Kebes und Simmias, daß Philolaos in ihrer Stadt einige Zeit lehrend verbracht habe. Demnach war er annähernd ein Zeitgenosse des Sokrates.

\section{Quellen}

D.L. VIII,84-85; Platon, Phaidon 6re; weitere Quellen bei DK 44 A. 
Werke

Ein einziges Buch, aus dem von den 26 bezeugten Fragmenten etwa zehn vermutlich echte Fragmente stammen (DK 44 BI-6, 6a, 7, I3, I7). Von dem sonstigen Material gehört viel der Überlieferung pseudo-pythagoreischer Schriften an, die in der späteren Antike verfaßt wurden (siehe Thesleff [I99]).

\section{Prodikos}

Geboren in der I. Hälfte des 5. Jahrhunderts v.Chr. auf der Kykladen-Insel Keos; ein Sophist, bekannt besonders wegen seiner Untersuchungen zur Sprache und auch wegen seiner fiktionalen Schrift Die Wahl des Herakles, in der der Held vor die Wahl gestellt wird, zwischen Tugend und Laster zu wählen, wobei diese als zwei sehr gegensätzliche Frauen auftraten (Xenophon, Mem. II,I,2I-34). Prodikos leitete den Ursprung der griechischen Götter und überhaupt der Religion daraus her, daß frühere Völker Dinge personifiziert hätten, von denen das Leben abhängt, zum Beispiel das Brot (Demeter) und den Wein (Dionysos); wie dem Protagoras, so wird auch ihm die Behauptung zugeschrieben, daß es nicht möglich sei, zu widersprechen (siehe Kerferd [433] 89-90). Gestorben ist er wahrscheinlich in den ersten Jahren des 4. Jahrhunderts v.Chr.

\section{Quellen}

Xenophon (wie oben angegeben); Platon, besonders Prot. 337a-c, Euthydem 277e; Philostratos, VS V,I2; außerdem weitere Quellen bei DK 84 A.

\section{Werke}

Jahreszeiten, ein Werk zur Ökonomie (aus dem das Exzerpt über Herakles stammt); eine Abhandlung Über die Natur des Menschen; wahrscheinlich eine Schrift Über die Richtigkeit der Namen sowie weitere nicht bezeugte Schriften.

\section{Protagoras}

Geboren ca. 485 v.Chr. in Abdera; wahrscheinlich der erste Grieche, der sich selbst einen Sophisten nannte, und derjenige, dessen einflußreiche Karriere dieses Berufsbild zum Ausdruck bringt; am bekanntesten wegen seines Relativismus und Agnostizismus. Bei Besuchen in Athen wurde Protagoras ein enger Freund des Perikles, und man bat ihn, Thurioi, der neuen Athener Kolonie in Sizilien, eine Verfassung und Gesetze zu geben. Die Überlieferung, daß er in Athen vor Gericht stand und wegen Gottlosigkeit verurteilt wurde, ist so gut wie sicher eine Erfindung. Er starb ca. 4I 5 v.Chr. 


\section{Quellen}

Protagoras ist das namentlich genannte Thema eines der größeren Dialoge Platons, und er spielt auch in Platons Theaitet eine bedeutende Rolle. Andere Quellen sind: D.L. IX,50-56; Philostratos, VS I, Io; Platon, Prot. passim; viele andere Quellen bei DK 8o A.

\section{Werke}

Diogenes Laertius bietet einen Katalog der Schriften des Protagoras (IX,55), von denen einige wahrscheinlich unecht oder durch Unterteilung einzelner Werke zustandegekommen sind. $\mathrm{Zu}$ seinen authentischen Abhandlungen gehören Über die Wahrheit, auch unter dem Titel Die Umstürzler bekannt (sie begann mit: "Aller Dinge Maß ist der Mensch«; BI), Pro- und Contra-Reden (Antilogiai) und Über die Götter, welche mit dem Satz anfing: »Über die Götter habe ich keine Möglichkeit, etwas zu wissen, weder daß sie sind noch daß sie nicht sind noch wie sie etwa an Gestalt sind«; B4). Erhalten sind uns annähernd zwölf kurze Fragmente. Zu seinen Beiträgen zur Literaturkritik und Sprachtheorie siehe D.L. IX,52-54; DK 80 A27-30.

\section{Pythagoras}

Geboren etwa 570 v.Chr. auf Samos, wanderte er ca. 530 nach Kroton in Süditalien aus, wo er »den dort angesiedelten Griechen Gesetze gab« (D.L. VIII,3) und eine Sekte gründete, die sich durch ihre rituellen Gebräuche auszeichnete, ferner durch ihre Verpflichtung auf ein "reines" Leben und durch eine Art gemeinschaftlichen Lebens. Pythagoras wurde idealisiert als ein "göttlicher Mann« mit Weisheit aus Ägypten und weiter aus dem Osten sowie mit übernatürlichen Kräften wie etwa der Kraft, sich an seine frühere Inkarnation zu erinnern. Bei den Neuplatonikern wurde er zum Gegenstand hagiographischer Lebensbeschreibungen. Unsicher ist, wieweit er, wenn überhaupt, die mathematischen und musischen Studien in Gang brachte, mit denen der Pythagoreismus hernach in Verbindung gebracht wurde. Er starb etwa 490 v.Chr.

Quellen

Siehe Huffman, in diesem Band S. 6I-64.

\section{Thales}

Geboren ungefähr 624 v.Chr. in Milet, nach Aristoteles (Metaph. I,3, 983b2off.) der erste Naturforscher und anderweitig als einer der sieben Weisen idealisiert und wie sie als ein Vorbild an politischer Weisheit. Herodot (I,I70) rühmte Thales wegen seines Rats an die jonischen Staaten, sich angesichts der Bedrohung durch Persien zu vereinigen. Er wird auch als der einzige Weise be- 
schrieben, der über den praktischen Bereich hinausgegangen sei (Plutarch, Solon 3-5), wie in der Anekdote, daß er in den Himmel schaute und dabei in einen Graben fiel; die Anekdote wird von Platon in einem Zusammenhang erzählt, wo Thales als der paradigmatische Philosoph vorgestellt wird (Tht. I74a-b). Thales soll eine Sonnenfinsternis vorausgesagt haben (wahrscheinlich 585 v.Chr.) und ein Fachmann im Ingenieurswesen, in der Geometrie und in der Astronomie gewesen sein und hat dieses Wissen möglicherweise bei einer Reise nach Ägypten gewonnen. Gestorben ist er etwa 546 v.Chr.

\section{Quellen}

D.L. I,22-44; Herodot I,74-75, I70; weitere Quellen bei DK I I A.

\section{Werke}

Die Quellen des Diogenes Laertius berichten, daß Thales nichts Schriftliches hinterlassen habe und daß eine ihm zugeschriebene Sternkunde für Seefahrer unecht sei $(I, 23)$.

\section{Xenophanes}

Geboren ca. 570 v.Chr. in Kolophon auf dem jonischen Festland. Nach der Eroberung Lydiens durch Persien im Jahr 545 führte Xenophanes ein Wanderleben mit verschiedenen Aufenthalten in sizilischen Städten. Nach seinem eigenen Zeugnis (DK 2 I B8) lebte er noch mit 92 Jahren. Die Verse, die uns von ihm erhalten sind, handeln von Kosmologie und Theologie, kritisieren konventionelle Werte, deuten einen kulturellen Relativismus und Skeptizismus an und und schließen auch traditionelle Themen poetischer Trinklieder ein. In der späteren Antike wurde Xenophanes als der Begründer der eleatischen Philosophie und als der Lehrer des Parmenides angesehen; aber wenn er Parmenides auch fast mit Sicherheit beeinflußt hat, sollte diese Überlieferung nicht ganz für bare Münze genommen werden.

\section{Quellen}

DK 2 I BI-3, B8; D.L. IX, I8-2I; Klemens von Alexandrien, Stromateis I,64; Platon, Soph. 242d; Aristoteles, Metaph. I,5, 986bI8, und in der pseudo-aristotelischen Schrift Über Melissos, Xenophanes, Gorgias (DK 30 A5); weitere Quellen bei DK 2 I A.

\section{Werke}

Ungefähr I 20 Verse sind erhalten. Mehr als die Hälfte sind elegische Verse; und es liegt ein elegisches Gedicht vor (BI), welches vollständig sein könnte. Die anderen Verse sind - abgesehen von einem jambischen Trimeter (BI4,I) Hexameter. Einige davon stammen aus den Silloi (satirische Verse) oder Parodien; in der Spätantike wurden Xenophanes mindestens fünf Bücher Silloi zu- 
geschrieben (B2 Ia). Proklos sagt, sie seien "gegen alle Philosophen und Dichter» gerichtet gewesen (DK 2 I A22; vgl. D.L. IX, I 8). Die übrigen erhaltenen Zeilen stammen möglicherweise ebenfalls aus diesem Werk, wenngleich bestimmte Fragmente zu einem Gedicht gehören könnten, das in hellenistischer Zeit den Titel Über die Natur trug. Darüber hinaus soll Xenophanes 2000 Verse über die Stadtgründungen von Kolophon und Elea geschrieben haben (D.L. IX,20).

\section{Zenon}

Geboren etwa 490 v.Chr. in Elea in Süditalien, wo er bei Parmenides studierte (Platon, Parm. I27a-b). Zenon ist der Verfasser von Paradoxien zur Unmöglichkeit von Bewegung und Vielheit. Diese Paradoxien werden im allgemeinen als eine Verteidigung des eleatischen Monismus aufgefaßt (siehe indes McKirahan, in diesem Band S. I22f.). Geschichten, daß er "etwa vierzig Jahre alt, wohlgewachsen und von angenehmem Aussehen", zusammen mit dem älteren Parmenides in Athen zu Besuch gewesen sei und dort Sokrates getroffen habe (Platon a.a.O.), könnten ebenso fiktiv sein, wie es die Chronologie des angenommenen Treffens sein muß (siehe Mansfeld [32] 64-68). Wie lange Zenon gelebt hat, läßt sich unmöglich bestimmen; aber sein Werk war so gut wie sicher Demokrit und vielleicht auch Anaxagoras bekannt.

\section{Quellen}

D.L. IX,25-29; die Suda; weitere Quellen bei DK 29 A.

Werke

Zenon wird wohl nur ein einziges Werk geschrieben haben, die »Schrift», die er nach der Darstellung Platons dem jungen Sokrates vorliest (Parm. I27c). 


\section{Chronologie}

Diese Liste früher griechischer Philosophen stellt in groben Zügen dar, wer Zeitgenosse von wem war. Angefügt ist eine zweite Liste mit prominenten Personen, die in diesem Buch erwähnt werden. Die meisten Daten gelten nur annäherungsweise und könnten nach vorne und hinten um Io oder 20 Jahre schwanken. Homer wird üblicherweise ins 8. Jahrhundert v.Chr. datiert, Hesiod ins 8. oder ins frühe 7. Jahrhundert.

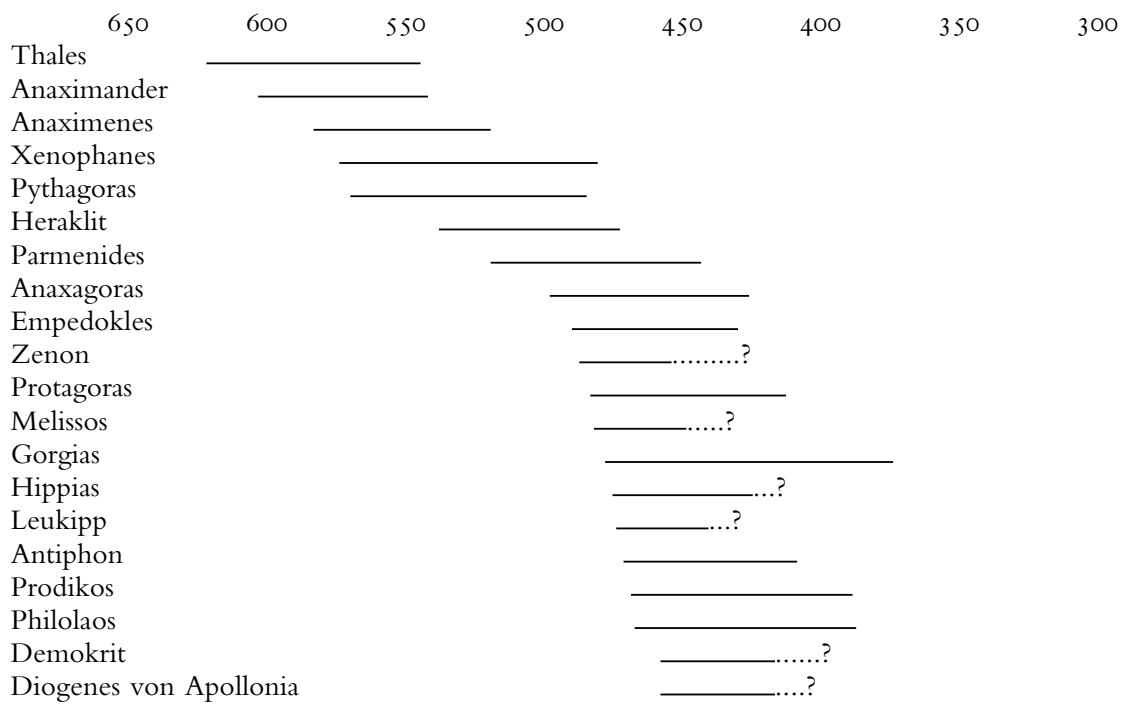

Andere Personen

Pherekydes

Hekataios

Herodot

Sokrates

Hippokrates

Thukydides

Platon

Archytas

Aristoteles

Theophrast

650

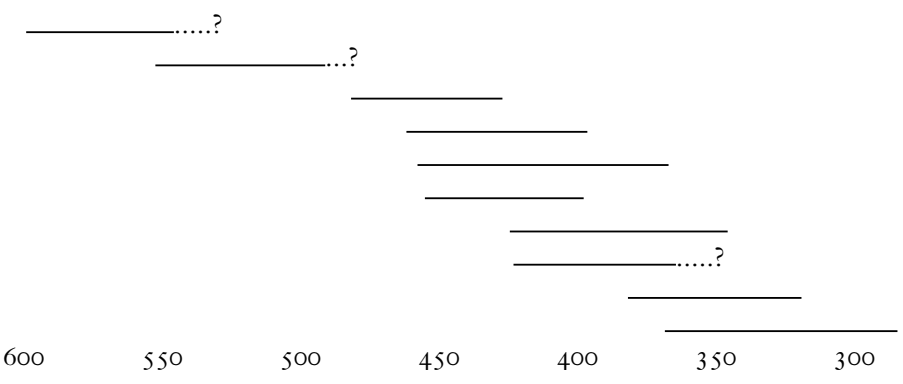




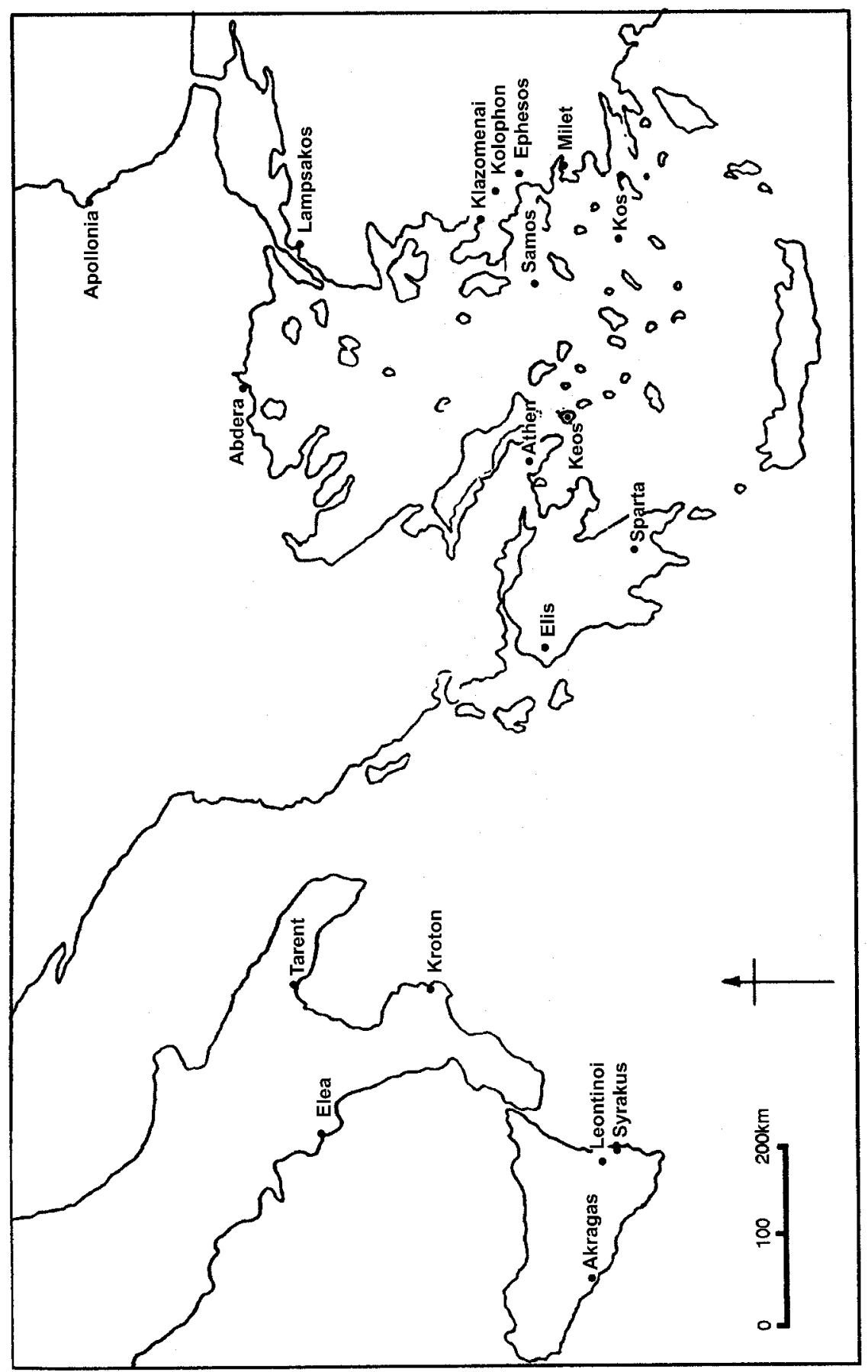

